\title{
The impact of diabetes process and outcome quality measures on overall survival in patients with co-morbid colorectal cancer
}

\author{
Elizabeth Y. Chiao, \\ Houston Health Services Research and Development Center of Excellence, Michael E. DeBakey \\ Veterans Affairs Medical Center, Houston, TX, USA. Department of Medicine, Baylor College of \\ Medicine, Houston, TX, USA. Houston Center for Quality of Care and Utilization Studies, Michael \\ E. DeBakey VA Medical Center (152), 2002 Holcombe Boulevard, Houston, TX 77030, USA
}

Preethi V. Nambi, and

Houston Health Services Research and Development Center of Excellence, Michael E. DeBakey Veterans Affairs Medical Center, Houston, TX, USA. Department of Medicine, Baylor College of Medicine, Houston, TX, USA

\begin{abstract}
Aanand D. Naik
Houston Health Services Research and Development Center of Excellence, Michael E. DeBakey Veterans Affairs Medical Center, Houston, TX, USA. Department of Medicine, Baylor College of Medicine, Houston, TX, USA
\end{abstract}

Elizabeth Y. Chiao: echiao@bcm.edu

\begin{abstract}
Purpose-To evaluate the effect of prevalent diabetes mellitus (DM) and quality of diabetes process and outcome measures on overall survival in colorectal cancer (CRC) patients.
\end{abstract}

\begin{abstract}
Patient and Methods-We conducted a retrospective cohort study among patients newly diagnosed with CRC. Kaplan-Meier analyses and Cox proportional hazard regression models were performed. Quality of diabetes process and outcomes measures in the year prior to CRC diagnosis were compared with those in the year after CRC diagnosis.
\end{abstract}

\begin{abstract}
Results-Four hundred and seventy CRC patients were identified during the study period, including $122(26 \%)$ patients with DM. Survival between diabetic and non-diabetic were not significantly different after stratifying by stage. In a multivariable model, only age (HR 1.04), stage 2 and 3 (HR 1.88), stage 4 (HR 4.26), and Deyo comorbidity score (HR 1.14) were significantly associated with increased risk of death. Overall, patients in this CRC cohort with DM had good to excellent diabetes quality of care in the year prior to diagnosis as evidenced by primary care and eye clinic visits, number of times diabetes intermediate outcomes were

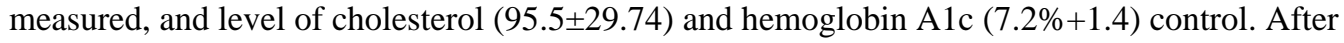
CRC diagnosis, there was no significant change in these quality of care indices compared to the year prior.
\end{abstract}

Conclusions-Prevalent DM did not affect overall survival in this cohort of VA patients diagnosed with CRC. The quality of diabetes care prior to CRC diagnosis, which persisted after diagnosis, may have moderated the mortality effect of diabetes in this CRC cohort.

\footnotetext{
(c) Springer Science+Business Media, LLC 2010

Correspondence to: Elizabeth Y. Chiao, echiao@bcm. edu.
} 


\section{Keywords}

Colorectal cancer; Diabetes mellitus; Survival; Quality of care; Outcomes

\section{Introduction}

Mortality from colorectal cancer (CRC) has been decreasing in the past 20 years due to advances in both early detection and treatment [1]. Thus, as individuals diagnosed with CRC have improved cancer-specific mortality, reducing all-cause mortality for co-morbid patients with CRC has become increasingly important [2]. In addition, the majority of new CRC diagnoses occur in older individuals, with more than two-thirds diagnosed in those over the age of 65 years [3, 4]. Because diabetes mellitus (DM) is one of the most common conditions among older Americans, DM is a common co-morbid medical condition among older individuals with CRC. As a result, there has been increasing scientific interest in the effect of DM on CRC incidence and survival. However, few studies have evaluated the impact of diabetes care quality on overall mortality among patients with co-morbid CRC.

Recent case-control [5, 6] and cohort studies [7-9] have shown that DM is associated with an increased risk of CRC. Furthermore, it also appears that DM is correlated with decreased CRC survival after diagnosis [2, 10]. Recently, a meta-analysis by Barone et al. [11] found that the pooled hazard ratios of long-term, all-cause mortality in CRC patients with prevalent DM were $32 \%$ higher than those without DM. In addition, poorly controlled DM (defined as hemoglobin A1C $>7.5 \%$ ) has been shown to increase mortality risk among CRC patients [12].

One recent study compared the quality of DM care delivered to diabetic patients with and without a history of cancer [13]. Although they were unable to determine if quality of DM care impacted survival after cancer diagnosis, they found that diabetic patients with a diagnosis of cancer had higher adjusted rates of Hemoglobin A1c (HbA1c) testing, and $\mathrm{HbA1c}$ control. They hypothesize that although there was the potential for cancer care services to compete with DM care, in their integrated health care system, the quality of DM patients with and without cancer were similar. Because the Veterans Administration (VA) healthcare system is also a large integrated health care system with an electronic medical record, we sought to use established diabetes care process and outcome measures to determine the impact of CRC diagnosis on the quality of DM care and specifically evaluate the potentially explanatory role of diabetes care, post CRC-diagnosis, on overall survival in this cohort.

\section{Patients and methods}

\section{Study cohort}

We searched the electronic pathology database of the Michael E. Debakey Veteran's Affairs Medical Center (MEDVAMC) in Houston, TX for patients with new, tissue-based diagnoses of colorectal cancer diagnoses between January 1999 and December 2006. The methods have been previously described in detail [14]. Briefly, the MEDVAMC serves approximately 116,000 veterans living in the Western Gulf-coast region, and is one of the largest VA health centers in the national VA healthcare system. Consecutive patients were identified and were excluded if: 1) patients did not obtain previous care at MEDVAMC(i.e., patients who did not have at least two outpatient visits, or one hospitalization in the year preceding CRC diagnosis); 2) patients were diagnosed or treated at other facilities; and 3) patients who had a previous colorectal cancer diagnosis. The study was approved by the Institutional Review Board for Human Subject Research for Baylor College of Medicine. 


\section{Study design}

We conducted a retrospective cohort study in patients with and without a previous diagnosis of DM who were newly diagnosed with CRC. Patients were followed from their date of CRC diagnosis until date of death or January 1, 2007, whichever came first. Briefly, we used a standardized form to collect demographic data (age, race, gender, and marital status), clinical information (height, weight, BMI, and comorbidities), laboratory values (albumin), as well as CRC specific information which included TNM staging at time of diagnosis and treatment. The date of CRC diagnosis was determined as the date of histological confirmation of pathology. Staging information was obtained via chart review. Furthermore, we calculated an overall disease comorbidity score for each patient using a validated approach adapted for use in administrative databases [15]. We excluded the ICD-9 codes for DM from the comorbidity coding.

To identify deaths, we reviewed the electronic medical record, and linked our cohort to the VA Vital Status File to capture possible deaths that were not recorded in the medical record. The VA vital status file includes information from Beneficiary Identification Records Locator Subsystem (BIRLS) Death File, the Medicare Vital Status File, the Social Security Administration's Death Master File (SSA DMF), and the VHA Medical SAS Inpatient Datasets (MSID). The VA Vital Status File has been shown to match the National Death Index (NDI) date of death in over $90 \%$ of cases [16].

Diabetic patients were identified by ICD-9 code, a method validated within the VA database [17], and the diagnosis was verified by chart audit for the current study. For diabetic patients, we reviewed the electronic records and documented additional information from one year prior to the diagnosis of CRC and one year after the diagnosis of CRC. This information included: clinical data (height, weight, and systolic and diastolic blood pressure) and laboratory values (Hemoglobin A1c, total cholesterol, and LDL)

\section{Statistical analyses}

Demographic and clinical characteristics of patients with and without DM were compared by Student's t-test for continuous variables (age, comorbidity score, BMI, Albumin, and length of follow-up) and Chi-square tests for categorical variables (gender, race, year of CRC diagnosis, dichotomized co-morbidity score, CRC stage, and receipt of cancer treatment).

Kaplan-Meier estimates of survival among patients with CRC over the follow-up period were stratified by presence of DM for each stage of CRC, and log-rank test for differences in survival between these groups was calculated. We conducted adjusted and unadjusted Cox proportional hazard regression models to calculate Hazard Ratios for the study variables. The adjusted model covariates included gender, race, age, year of CRC diagnosis, staging, co-morbidity score, and treatment received. Wald statistics were calculated for the Cox model parameter estimates to assess the effect of these covariates. The model parameter estimates and standard errors were used to calculate adjusted hazard ratio estimates, and the 95\% Wald confidence intervals for these ratios. The proportional hazard assumption was fulfilled in all models.

To compare the diabetic care parameters for all diabetic patients 1 year pre- and 1 year postCRC diagnosis, Student's t-test statistics were calculated. All of the patients with DM were included in both the pre-CRC and post CRC-analyses, regardless of their date of death. Because the severity of CRC and terminal illness may impact some metabolic parameters for diabetics, we conducted several sensitivity analyses evaluating the effect of excluding the patients who died during the study period. The results of the sensitivity analyses did not change the findings. 


\section{Results}

\section{Study characteristics}

A total of 470 CRC patients were identified during the study period, including 122 (26\%) patients with DM. The median follow-up for the cohort was 35.2 months. There were 202 deaths (43\%). The median age of the cohort was 68 and the cohort was $99 \%$ male. The age, gender, race, year of diagnosis, and stage of diagnosis were not significantly different among those with diabetes compared to non-diabetics. Compared to non-diabetics, however, those with diabetes were significantly less likely to receive chemotherapy (38.1\% versus $24.8 \%, p=0.01)$ and radio-therapy $(18.5 \%$ versus $10.3 \%, p=0.04)$. Patients with diabetes also had significantly higher BMI $(26.0 \pm 5.6$ versus $29.6 \pm 7.19, p<0.01)$ and serum albumin levels ( $3.14 \pm 0.74$ versus $3.37 \pm 0.57, p=0.01)$ at the time of diagnosis. The mean follow-up time was similar between the two groups, with an overall period of 37.9 months \pm 26.5 months. The mortality rate between diabetics and those without diabetes was similar $(42.6 \%$ versus 48.3\%). See Table 1 for detailed results.

\section{Survival analyses}

Figures 1, 2, and 3 illustrate the Kaplan Meier survival curves comparing patients with DM to those without DM by staging categories. Survival was not significantly different between groups (using log-rank p-values) for each stage category: stage 0 or $1(p=0.78)$, stage 2 or 3 $(p=0.97)$, and stage $4(p=0.17)$. The median survival for patients with stage 0 or $1 \mathrm{CRC}$ and diabetes was 6.16 years, and the median survival for patients without DM was not reached during this study period. Median survival for patients diagnosed at stage 2 or 3 was 6.7 years among patients with DM and 5.9 years for non-diabetics. Finally, median survival for stage 4 patients was 3 years among those with DM, compared to 2 years among non-diabetics.

\section{Unadjusted and adjusted hazard ratios}

Table 2 shows the unadjusted and adjusted hazard ratios for death derived from Cox regression models for all 470 patients diagnosed with colon cancer. DM was not associated with death in either model. In the adjusted model, only age (HR 1.04, $p<0.01$ ), stage 2 and 3 (HR 1.88, $p 0.01$ ), stage 4 (HR 4.26, $p<0.01$ ), and Deyo co-morbidity score (HR 1.14, $p<0.01)$ were associated with increased risk of death. Having received radiation therapy (XRT) or chemotherapy was protective, but the p-value did not achieve significance $(p=0.38)$. Only surgical excision of CRC was significantly protective of death (HR 0.34 , $p<0.01)$.

\section{Quality of diabetes care before and after colorectal cancer diagnosis}

Measures of diabetes care were collected for all patients with diabetes for the twelve months preceding and following cancer diagnosis. These measures are broadly differentiated into intermediate outcome measures and process of care measures. In evaluating the outcome measures (top row in Table 3), the mean values for HbA1c and low density lipoprotein (LDL) cholesterol in the year before cancer diagnosis were at or near levels consistent with diabetes control ( $\leq 7 \%$ and $\leq 100 \mathrm{mg} / \mathrm{dl}$, respectively) and both significantly improved within the controlled range in the year after CRC diagnosis ( $7.2 \pm 1.4$ to $6.7 \pm 1.2, p=.02$; and $96 \pm 30$ to $86 \pm 31, p=.04$ ). Mean values for other intermediate outcomes measures of diabetic care (i.e., systolic and diastolic blood pressures and total cholesterol) all improved slightly in the year following CRC diagnosis but were not statistically significant.

Table 3 also displays (bottom row) a comparison of the diabetes process of care measures in the years prior to and following CRC diagnosis. These measures include the frequency of primary care and eye clinic visits and the mean number of laboratory checks per person for the intermediate outcome measures (systolic and diastolic blood pressure, hemoglobin A1C, 
and LDL cholesterol). Most patients had multiple visits to their non-oncology, primary care providers in the year after cancer diagnosis. The mean number of primary care and eye clinic visits were not significantly different between the year prior to and the year following CRC diagnosis. Similarly, the mean number of blood pressure and hemoglobin A1c measurements did not significantly change. However, the mean number of LDL cholesterol measurements did significantly decline in the year after CRC diagnosis $(1.39 \pm 1.0$ versus $1.05 \pm 1.0, p=0.01$ ), although, most patients still had at least one LDL cholesterol check in the year following cancer diagnosis.

\section{Discussion}

This retrospective review of newly diagnosed CRC patients from a regional VA medical center is among the first to evaluate all-cause mortality after a new diagnosis of CRC in patients with prevalent DM in the context of confirming DM quality of care indicators. In a multivariable model, we found that patients with co-morbid DM had similar survival at all CRC stages compared to those without diabetes. Furthermore, we found that at baseline, this group of diabetics demonstrated excellent quality of DM care, measured by: Hemoglobin A1c, total cholesterol measurements, and primary care and eye clinic visits. We also found that this level of care remained relatively unchanged after CRC diagnosis. We hypothesize that the quality of DM care before and after CRC diagnosis positively impacted survival among diabetics.

Although the association between CRC-specific mortality and DM is somewhat equivocal $[10,12,18,19]$, recent cohort and case-control studies, and a meta-analysis have shown that patients with pre-existing DM and CRC have higher all-cause mortality compared to patients without diabetes. While differentiating between cancer-specific and all-cause mortality can be challenging, these findings suggests that the cause of increased mortality among DM patients with CRC may be related more to complications specifically related to DM. Thus, improved diabetes care during and after cancer diagnosis may have meaningful impact on survival in diabetic patients with cancer.

In this cohort of patients who received routine primary care within an integrated health system, we found that the quality of DM care was good to excellent prior to CRC diagnosis evidenced by number of primary care visits and mean levels for Hemoglobin A1c and LDL cholesterol. In addition, because we limited our cohort to individuals who had previously received primary care in the system, our population was likely adherent to medical care and treatments related to their DM, as evidenced by the fact that median Hemoglobin A1C of the cohort 1 year prior to CRC diagnosis was 7.1\%.

Furthermore, the quality of diabetes care in the year after CRC diagnosis remained good, which may have been a result of ongoing primary care visits after cancer diagnosis. The advantages of an integrated system like the VA (e.g., access to routine primary and specialty care, linkage of treatment planning by electronic medical records, computerized alerts, audit/ feedback of provider performance, and availability of on-site laboratory and pharmacy services), $[20,21]$ may contribute to our finding that high quality of diabetes care was largely unchanged after CRC diagnosis. We hypothesize that the availability and quality of primary care prior to cancer diagnosis and the persistence of quality diabetes care after CRC diagnosis, may partially explain why there was no difference in overall survival between patients with and without prevalent diabetes in this cohort.

In addition, improved communication between primary care physicians and oncologists identified patients at high risk for chemotherapy-related toxicities, and subsequently excess chemotherapy toxicity was avoided. It has been shown that patients with preexisting 
diabetes have poorer responses to cancer treatments, and may be more likely to have increased chemotherapy-related toxicities compared with non-diabetic patients who are receiving chemotherapy [11]. In our cohort, diabetic patients were significantly less likely to receive chemotherapy and radiation therapy; therefore, morbidity and mortality associated with chemotherapy and radiation therapy treatment may have been diminished.

The strengths of the current study include our ability to assess laboratory data, including Hemoglobin A1c and LDL cholesterol, as well as quality indicators (such as frequency of primary care clinic visits, and eye clinic visits) associated with diabetic care in the year preceding and the year after CRC diagnosis. In this way, we were able to determine the quality of diabetes care among the diabetics in the cohort. In addition, through the vital records file and electronic medical records, mortality data have been validated and have been shown to be extremely accurate. Finally, because we utilized a cohort assembled from a single, integrated healthcare center, the evaluation and treatments for CRC and diabetes were likely standardized and relatively homogenous due to a stable group of treating physicians. Therefore, bias associated with treatment variation due to small area (geographic) variations based on physician preference was diminished [22].

Our study has several limitations. First, the sample size of our CRC and diabetic patients is smaller than many large database studies. Because we adjusted for several variables including a comorbidity, we may not have the power to detect small differences in survival between CRC patients with and without diabetes. Second, we do not know the etiology of the improvement in Hemoglobin A1c and LDL cholesterol one-year post CRC diagnosis. It is possible that these values improved because of weight loss related to the cancer or its treatment. However, our findings that the number of primary care visits and eye visits before and after CRC diagnosis, as well as the number of systolic blood pressure, diastolic blood pressure, hemoglobin A1c, and cholesterol checks did not change after CRC diagnosis suggest that primary care providers remained vigilant regarding diabetes care after CRC diagnosis, implying that these patients were not terminally ill. Finally, our study was conducted in a VA facility, and our cohort was nearly all male. In addition, we limited our cohort to individuals who had previous evidence of care at the VA facility. Thus, our findings may have limited applicability outside the VA. However, this study does highlight ways in which regular use of services from an integrated healthcare system such as the VA among patients with cancer and other common co-morbidities may significantly impact mortality after cancer diagnosis.

In summary, we did not detect an increase in all-cause mortality associated with having a prevalent diagnosis of DM. Our findings suggest that the quality of diabetes care around the time of, but particularly after cancer diagnosis may impact overall mortality among those with co-morbid diabetes. The inherent strengths of an integrated health care system, and patient adherence to these services may partially explain our findings via improved care coordination between primary care providers and specialists using tools like an electronic medical record. Based on our findings, it may be important for primary care physicians and cancer care specialists to better coordinate complex, co-morbid cancer care, to optimize diabetes control. It will be important in the future to determine if these results can be prospectively verified in other large health care systems, and to determine if interventions to improve communication between primary care physicians and oncologists can impact the mortality rates of patients with co-morbid diabetes and cancer.

\section{Acknowledgments}

This article is the result of work supported with resources and the use of facilities at the Houston Health Services Research and Development Center of Excellence (HFP90-020), Michael E. DeBakey Veterans Affairs Medical Center and by a pilot grant from the Dan L. Duncan Cancer Center at Baylor College of Medicine, titled "Role of 
co-morbidity and Frailty in Older Adults Diagnosed with Colorectal Cancer." Dr. Chiao received support from the National Cancer Institute (K23CA124318) and Dr. Naik received support from the National Institute of Aging (K23AG027144) and the Doris Duke Charitable Foundation.

The authors would like to thank and acknowledge Annette Walder, MS, for her assistance with data analysis, and Hashem El-Serag, MD, MPH and Hardeep Singh, MD, MPH for access to the colorectal cancer data at the Michael E. DeBakey VA Medical Center from which the current analyses were based.

Funding Support This work was supported in part by the Houston VA HSR\&D Center of Excellence (HFP90-020) at the Michael E. DeBakey VA Medical Center and a pilot grant from the Dan L. Duncan Cancer CenterPrevention and Population Sciences Program at the Baylor College of Medicine. Dr. Chiao is also supported by a National Cancer Institute K23 grant (K23CA124318). Dr. Naik is also supported by a National Institute on Aging K23 grant (5K23AG027144) and a Doris Duke Charitable Foundation Clinical Scientist Development Award. No funding agencies had a role in the design and conduct of the study, analysis and interpretation of data, or preparation and approval of the manuscript. The views expressed herein are those of the authors and do not necessarily reflect those of the Department of Veterans Affairs or Baylor College of Medicine.

\section{References}

1. American Cancer Society. Colorectal Cancer Facts and Figures 2008-2010. 2009 Ref Type: Report.

2. Gross CP, Guo Z, McAvay GJ, Allore HG, Young M, Tinetti ME. Multimorbidity and survival in older persons with colorectal cancer. J Am Geriatr Soc. 2006; 54:1898-904. [PubMed: 17198496]

3. Yancik R. Comorbidity and age as predictors of risk for early mortality of male and female colon carcinoma patients: a population-based study. 1998

4. Ries LAG, Melbert D, Krapcho M, Stinchcomb DG, Howlader N, Horner MJ, Mariotto A, Miller BA, Feuer EJ, Altekruse SF, Lewis DR, Clegg L, Eisner MP, Reichman M, Edwards BK. SEER Cancer Statistics Review, 1975-2005. 2007 Ref Type: Electronic Citation.

5. Williams JC. Colon carcinoma and diabetes mellitus. 1984

6. O’Mara BA. Diabetes mellitus and cancer risk: a multisite case-control study. 1985

7. La VC. Diabetes mellitus and colorectal cancer risk. 1997

8. La VC. History of selected diseases and the risk of colorectal cancer. 1991

9. Nilsen TI. Prospective study of colorectal cancer risk and physical activity, diabetes, blood glucose and BMI: exploring the hyper-insulinaemia hypothesis. 2001

10. Meyerhardt JA, Catalano PJ, Haller DG, Mayer RJ, Macdonald JS, Benson AB III, et al. Impact of diabetes mellitus on outcomes in patients with colon cancer. J Clin Oncol. 2003; 21:433-40. [PubMed: 12560431]

11. Barone BB, Yeh HC, Snyder CF, Peairs KS, Stein KB, Derr RL, et al. Long-term all-cause mortality in cancer patients with preexisting diabetes mellitus: a systematic review and metaanalysis. JAMA. 2008; 300:2754-64. [PubMed: 19088353]

12. Siddiqui AA, Spechler SJ, Huerta S, Dredar S, Little BB, Cryer B. Elevated HbA1c is an independent predictor of aggressive clinical behavior in patients with colorectal cancer: a casecontrol study. Dig Dis Sci. 2008; 53:2486-94. [PubMed: 18409001]

13. Keating NL. Quality of diabetes care among cancer survivors with diabetes. 2007

14. Wattacheril J. Lagtimes in diagnosis and treatment of colorectal cancer: determinants and association with cancer stage and survival. 2008

15. Deyo RA. Adapting a clinical comorbidity index for use with ICD-9-CM administrative databases. 1992

16. Arnold N, Sohn M, Maynard C, Hynes DM. VIReC Technical Report 2: VANDI Mortality Data Merge Project. Apr 9.2006

17. El-Serag HB. Statins are associated with a reduced risk of hepatocellular carcinoma in a large cohort of patients with diabetes. 2009

18. Polednak AP. Comorbid diabetes mellitus and risk of death after diagnosis of colorectal cancer: a population-based study. Cancer Detect Prev. 2006; 30:466-72. [PubMed: 17069990]

19. Shonka NA, Anderson JR, Panwalkar AW, Reed EC, Steen PD, Ganti AK. Effect of diabetes mellitus on the epidemiology and outcomes of colon cancer. Med Oncol. 2006; 23:515-9. [PubMed: 17303910] 
20. Kerr EA. Making performance indicators work: experiences of US Veterans Health Administration. 2007

21. Jha AK. Effect of the transformation of the Veterans Affairs Health Care System on the quality of care. 2003

22. Nattinger AB. Geographic variation in the use of breast-conserving treatment for breast cancer. 1992 


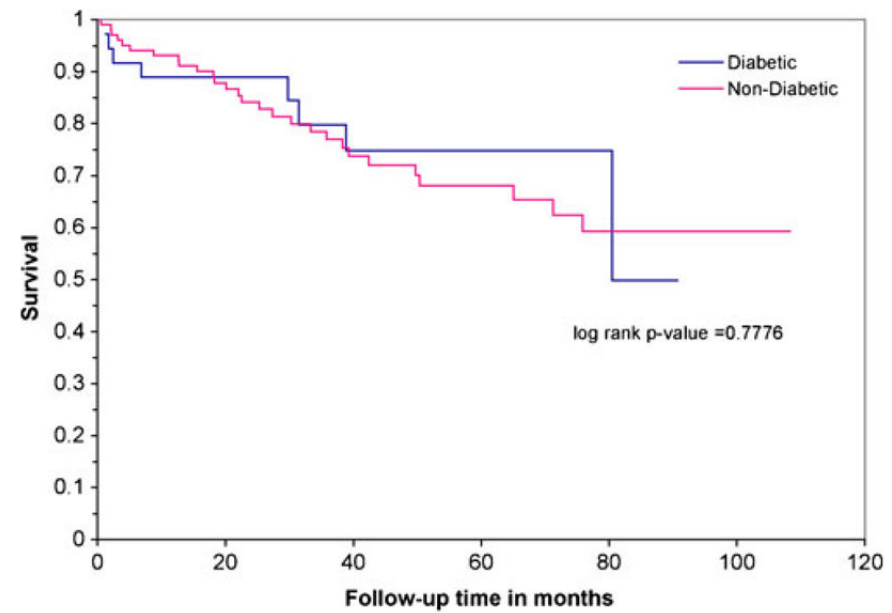

FIGURE 1.

Kaplan Meier survival analysis of 137 patients with stage 0,1 colorectal cancer by preexisting diabetes mellitus. 


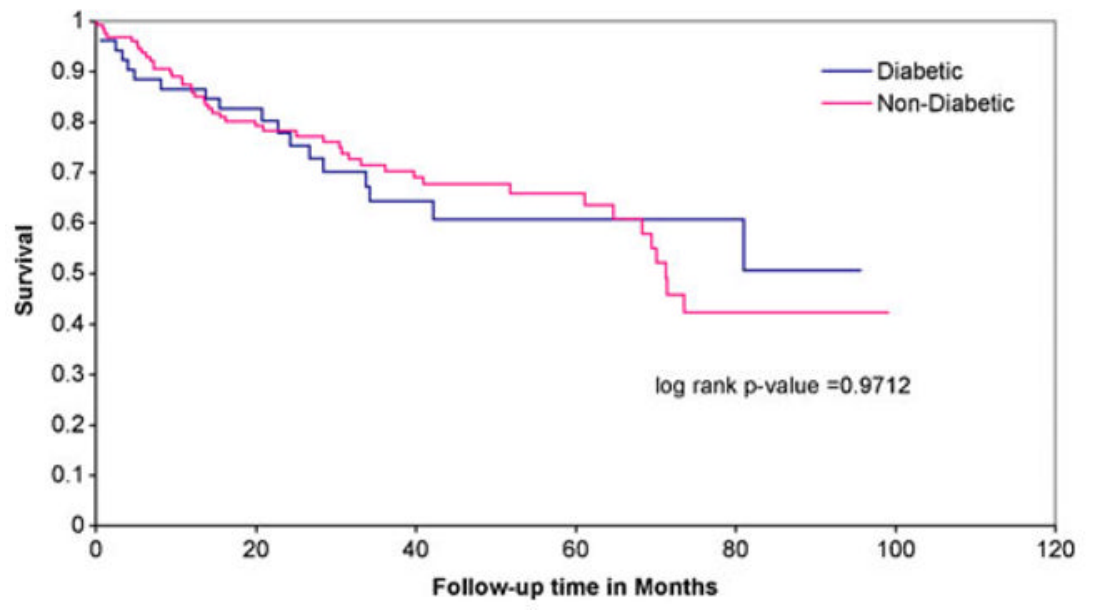

FIGURE 2.

Kaplan Meier survival analysis of 179 patients with stage 2,3 colorectal cancer by preexisting diabetes mellitus. 


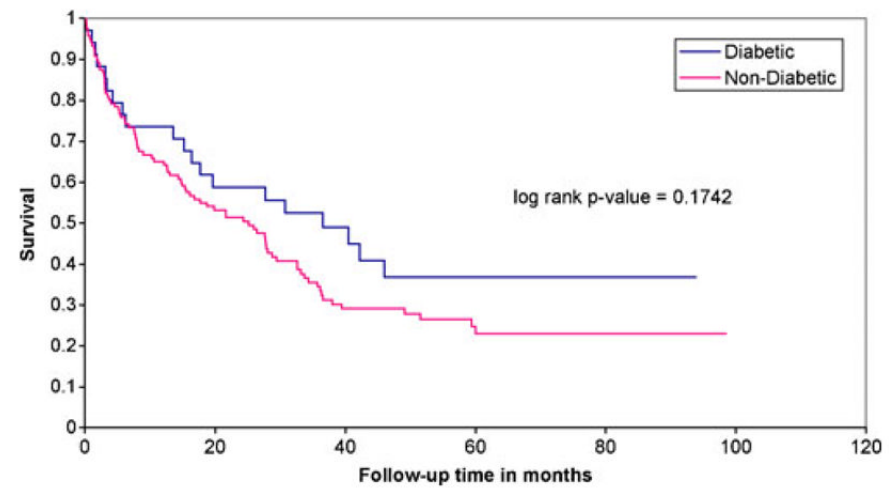

FIGURE 3.

Kaplan Meier survival analysis of 154 patients with stage 4 colorectal cancer by pre-existing diabetes mellitus. 
Table 1

Study characteristics of 470 colon cancer patients

\begin{tabular}{|c|c|c|c|}
\hline Characteristics & Diabetics $(n=122)$ & Non-Diabetics $(n=348)$ & $p$-value \\
\hline Age, mean (years) $\pm \mathrm{SD}$ & $67.7 \pm 9.7$ & $67.7 \pm 10.1$ & .97 \\
\hline Male gender, $\mathrm{n}(\%)$ & $122(100)$ & $342(98.3)$ & .14 \\
\hline Race, n (\%) & & & .90 \\
\hline African American & $37(30.3)$ & $97(27.9)$ & \\
\hline Hispanic & $11(9.1)$ & $28(8.1)$ & \\
\hline White & $60(49.6)$ & $181(52.0)$ & \\
\hline Others/Missing & 14 (11.6) & $42(12.1)$ & \\
\hline Year of CRC & & & .82 \\
\hline \multicolumn{4}{|l|}{ Diagnosis, n (\%) } \\
\hline 1999-2001 & $27(22.1)$ & $85(24.4)$ & \\
\hline $2002-2004$ & $50(41.0)$ & $144(41.4)$ & \\
\hline $2005-2006$ & $45(36.9)$ & $119(34.2)$ & \\
\hline Staging, n (\%) & & & .35 \\
\hline Stage 0,1 & $36(29.5)$ & $101(29.0)$ & \\
\hline Stage 2,3 & $52(42.6)$ & $127(36.5)$ & \\
\hline Stage 4 & $34(27.9)$ & $120(34.5)$ & \\
\hline \multicolumn{4}{|l|}{ Treatment received, $\mathrm{n}(\%)$} \\
\hline Radiation therapy & $12(10.3)$ & $63(18.5)$ & .04 \\
\hline Chemotherapy & $30(24.8)$ & $128(38.1)$ & .01 \\
\hline Surgery & $88(72.1)$ & $236(68.2)$ & .42 \\
\hline Modified Deyo co morbidity score, mean \pm SD & $4.28 \pm 2.84$ & $4.14 \pm 3.15$ & .66 \\
\hline $\mathrm{BMI}$, mean $\pm \mathrm{SD}$ & $29.6 \pm 7.19$ & $26.0 \pm 5.60$ & $<0.01$ \\
\hline Albumin, mean \pm SD & $3.37 \pm .57$ & $3.14 \pm .74$ & .01 \\
\hline Follow-up after diagnosis, mean (months) $\pm \mathrm{SD}$ & $35.51 \pm 25.94$ & $35.04 \pm 26.57$ & .86 \\
\hline Deaths, n (\%) & $52(42.62)$ & $168(48.28)$ & .28 \\
\hline
\end{tabular}

$\mathrm{SD}=$ standard deviation

$\mathrm{BMI}=$ body mass index

$\mathrm{CRC}=$ colorectal cancer 
. 
. 